\title{
Kinematics, control and workspace analysis of a bowden wire actuated manipulator for minimally invasive single-port surgery
}

\author{
S. Can, B. Jensen, E. Dean-Leon, C. Staub, A. Knoll \\ Robotics and Embedded Systems \\ Technical University Munich \\ $\{$ cans $\mid$ jensen $\mid$ dean | staub|knoll\}@in.tum.de
}

\author{
A. Fiolka, A. Schneider, A. Meining, H. Feussner \\ Klinikum rechts der Isar \\ Technical University Munich \\ \{fiolka|schneider|feussner\}@mitigroup.de
}

\begin{abstract}
This work presents the kinematics and control of a $6 \mathrm{DOF}$ manipulator for single-port surgery. Two such highly versatile manipulators and a semi-rigid telescope are combined in a unit that can be inserted through a $30 \mathrm{~mm}$ incision into the abdominal cavity. A velocity-based task-space control was implemented in a simulation environment to evaluate the kinematics of the manipulator and position control of the instruments. The manipulators are operated with two spacemouse that are integrated as a new input device. An evaluation of the system in a pick-and-place scenario is also presented. Finally, a workspace analysis was accomplished to assess the capabilities and drawbacks of the realized system.
\end{abstract}

\section{INTRODUCTION}

In practice laparoscopic surgery has proven itself to have numerous advantages over conventional surgical techniques for the patient such as diminished discomfort, reduced trauma, improved cosmetic outcomes and shorter hospitalization stays. Despite these benefits widespread application has been held up by several drawbacks. Primary among these is the limited flexibility, the restricted visual access and poor ergonomics. Investigations into new surgical procedures and techniques that could reduce trauma to the patient without compromising surgical ability are currently underway [1]. More research is still required to overcome the existing hurdles such as high costs, limited flexibility, and bulky designs. Currently there are two promising alternative surgical approaches, which are encouraging due to their enhanced technical possibilities: (i) Laparoscopic single-port surgery is performed through a single incision in the abdominal wall, where all the required instruments, usually up to four, are inserted. (ii) NOTES (Natural Orifice Transluminal Endoscopic Surgery) surgeries are performed through natural orifices and avoid an abdominal incision.

The topic of single port surgery has already been thoroughly discussed resulting in the publication of a consensus paper, which defined the challenges facing single-port surgery [2]. The aim is to have several independently controllable instruments and manipulators bundled together and introduced through a single point of access. The operational platform thus plays a central role for the advancement of such taxing interventions.

Laparoscopic single-port surgeries are currently carried out using manually articulated instruments that are introduced through trocar-like devices with three channels [3].
The SPIDER, developed by TransEnterix, is a passive, sterile and disposable single-port platform, which achieves an improved triangulation [4]. Kaouk et al. reported the first series of single-port robotic procedures on humans using the daVinci system [5]. Retraction and port related problems, triangulation, the crowding of instruments, and degraded visualization are limitations of the daVinci system that have yet to be conquered [6]. Intuitive Surgical is currently developing a new single-port manipulator [7], in addition to the development of new instruments for single-port interventions [8]. Another robot platform proposed for single- port surgery is the SPRINT [9]. This platform includes two 6 DOF robots, each with a diameter of $18 \mathrm{~mm}$ and integrated motors, and is introduced through a $30 \mathrm{~mm}$ incision. A new design proposed for single-port access surgery is described in IREP [10]. This platform design with 17 DOF in total is composed of two snake-like continuum robots and a stereo vision module introduced through a $15 \mathrm{~mm}$ incision.

We developed the novel Highly Versatile Single-Port System (HVSPS) in an attempt to address and overcome the existing drawbacks of single-port surgery. This paper details the implementation and evaluation of a task-space control, as well as the kinematics of the developed manipulators. A workspace analysis was likewise performed to evaluate the theoretical capabilities and effectiveness of the singleport system. The future concept of the platform is illustrated schematically in Fig. 1.

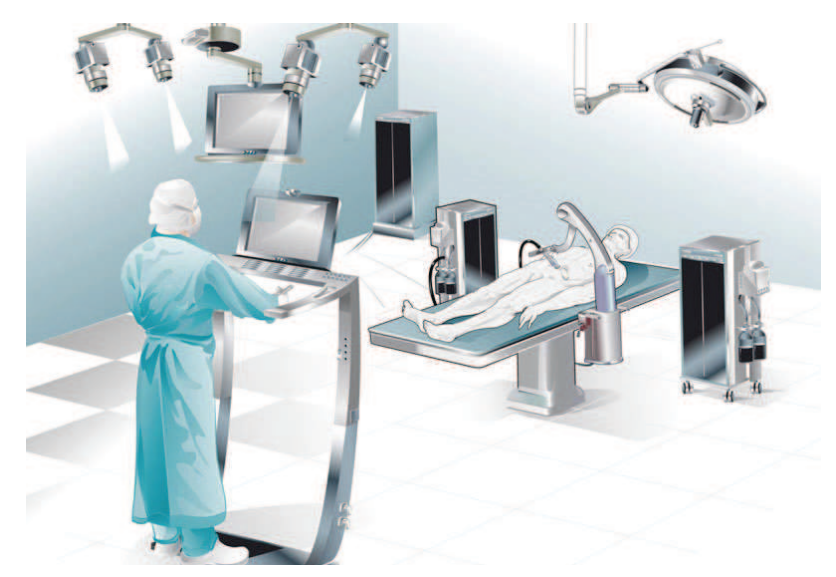

Fig. 1. Vision: The intuitive master console on the surgeon's side and the guiding manipulator with the single-port platform on the patient's side. 


\section{The Highly Versatile Single Port System}

The specifications and design of the developed single-port system was already published in [11]. This chapter briefly describes and summarize the current state of the entire system. The highly versatile single port system mainly consists of the following two units: on the one hand, as shown in Fig. 3, a multi-arm platform was developed that is inserted through a single access into the patient and remotely actuated by using a flexible drive transmission mechanism. On the other hand, a modular drive platform was developed that actuates all the articulations from $2 \mathrm{~m}$ at the periphery. The passively controlled, highly versatile single port platform consists of two articulated hollow manipulators with 6 DOFs and a semi-rigid telescope with 5 DOFs that are combined into a single unit. The bowden wire actuated manipulators and the telescope are integrated gas-tightly in the platform tube with a distal diameter of $30 \mathrm{~mm}$ and inserted together in a straight configuration through the trocar into the abdominal cavity. The platform tube is mounted on the proximal unit of the passive platform in which the linear and rotary articulations of the manipulators are implemented. An additional joint was developed to enable the rotation of the entire platform, which is mounted on the proximal tube.

Fig. 2 shows the developed single-port system during an in-vitro evaluation on the human mock-up ELITE trainer. The passive single-port platform is attached at the mounting flange to the hydraulic SoloAssist ${ }^{\mathrm{TM}}$ (Aktormed, Germany) telemanipulator with 3 DOFs that provides the guidance at the invariant point. This figure also shows the drive units of the manipulators in the background, on which the flexible hoses with the bowden wires are attached. The passive single-port platform has a distal diameter of $30 \mathrm{~mm}$, a proximal diameter of $100 \mathrm{~mm}$ and a total length of $400 \mathrm{~mm}$ from the SoloAssist link to the platform tip. The entire platform, including the rotation unit, has a total weight of $3 \mathrm{~kg}$. The working ranges of the manipulator joints is presented in Table I and the workspace of the instruments (manipulation range of the surgical intervention) is specified to be $100 \times 60 \times 60 \mathrm{~mm}$.

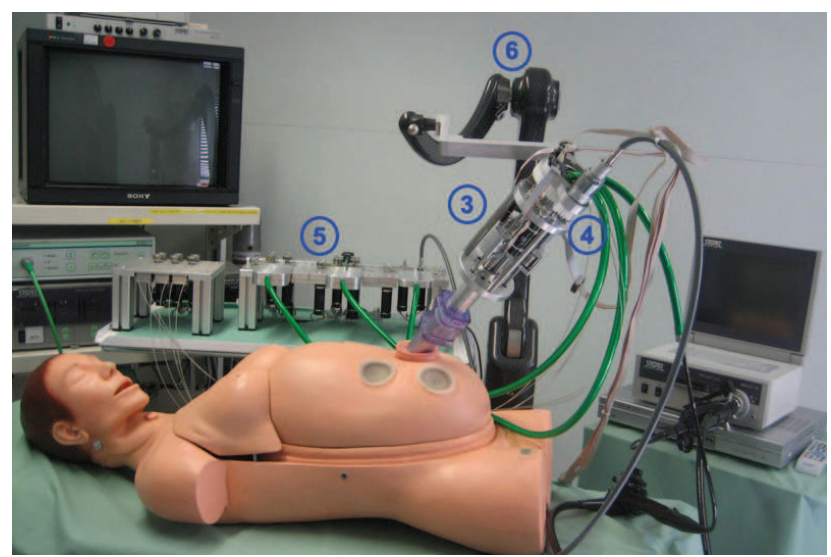

Fig. 2. The overall highly versatile single-port system: Passive platform attached to the SoloAssist guiding system and actuated by the active drive unit at the periphery.
TABLE I

OPERATING RANGE OF THE MANIPULATOR JOINTS

\begin{tabular}{|c|c|c|c|c|c|c|}
\hline & $\theta_{\mathbf{1}}$ & $d_{2}$ & $\theta_{\mathbf{3}}$ & $\theta_{\mathbf{4}}$ & $\theta_{\mathbf{5}}$ & $\theta_{\mathbf{6}}$ \\
\hline Workspace & $\pm 270^{\circ}$ & $80 \mathrm{~mm}$ & $\pm 90^{\circ}$ & $\pm 180^{\circ}$ & $\pm 150^{\circ}$ & $\pm 90^{\circ}$ \\
\hline
\end{tabular}

The entire HVSPS comprises the following components: (1) two manipulators with three distal articulations each, enabling an operation in opposed configuration, (2) a doublebending telescope with a large workspace for enhanced visualization, (3) a three-arm single-port platform with additional linear and rotary articulations integrated at the proximal end, (4) an articulation unit for rotation of the entire single-port platform, (5) a modular bowden wire actuation mechanism at the periphery and (6) a telemanipulator for guiding the passive platform at the access point.

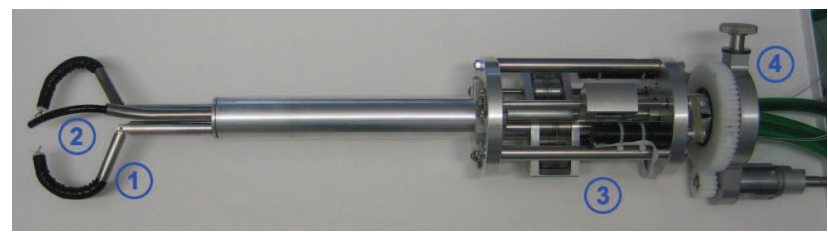

Fig. 3. The highly versatile single-port platform: Two 6 DOF manipulators $(\emptyset 12 \mathrm{~mm})$ and a 5 DOF semi-rigid telescope $(\emptyset 10 \mathrm{~mm})$ integrated into a platform unit with a distal diameter of $30 \mathrm{~mm}$.

Fig. 4 shows the distal section of the developed manipulators with the joints deflected to an opposed configuration. The bending section of the manipulator with 2 DOFs has a length of $75 \mathrm{~mm}$ followed by a $50 \mathrm{~mm}$ long tube and an "elbow" articulation. Two further DOFs at the proximal end of each manipulator provide a rotation of $\pm 270^{\circ}$ and a linear motion of $80 \mathrm{~mm}$ into the abdominal cavity. Differing from the other single-port systems, the developed hollow manipulators enable the insertion of flexible, endoscopic instruments that can be exchanged by an automated tool changer at the periphery. The distal rotation of the instruments represents the 6th DOF of the manipulators.

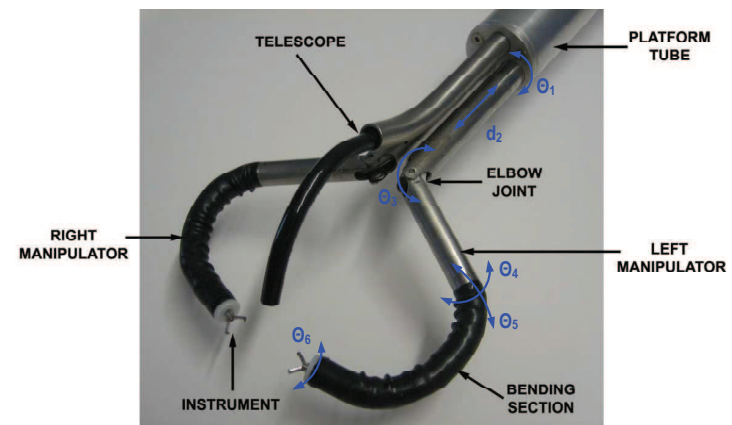

Fig. 4. Distal section of the single-port platform that is inserted into the abdominal cavity. All the joints of the manipulator are labeled.

Due to the lack of the designed double-bending telescope, which is under development, we decided to first realize the visualization by using a commercial endoscope with an outer diameter of $6 \mathrm{~mm}$ that is inserted through a $10 \mathrm{~mm}$ tube with a distal deflection of $30^{\circ}$ and controlled manually. 


\section{Kinematics of the Single-Port Manipulator}

We present here the forward and instantaneous kinematics of the manipulator that is used to realize a task-space control of the instruments. The kinematics of the manipulator with 6 DOFs in total, including the rotation of the instruments, is calculated using the kinematics of the 2 DOF bending section as published in [11].

Fig. 5a illustrates the implemented kinematics of the manipulator with the six joints. The kinematics of the bending section as shown in Fig. 5b is determined, first, relative to the base of the bending section $\{3\}$ and then mapped to the platform base $\{0\}$, which is placed at the middle of the distal tip of the platform. All the hinges of the bending section are considered as independent joints and reduced at the end to the joints $\theta_{4}$ and $\theta_{5}$ of the manipulator. The sum of all the partial angles $\vartheta_{i}$ (13 hinges), results in the joint $\theta_{4}$, and the sum of all the partial angles $\phi_{i}$ ( 6 hinges), in the joint $\theta_{5}$. The distal rotation of the flexible instruments is represented by $\theta_{6}$ and the rotation about the elbow joint is defined as $\theta_{3}$. The rotation $\theta_{1}$ and linear motion $d_{2}$ are the two proximal joints of the manipulator.

- $\{i\}$ : right handed frame with the axis $\left\{x_{i}, y_{i}, z_{i}\right\}$

- $p_{a}^{b}$ : position vector from point $a$ to $b$

- $T_{a}^{b}$ : transformation matrix from frame $a$ to $b$

- ${ }_{a} v_{i}^{\mathrm{e}},{ }_{a} w_{i}^{\mathrm{e}}$ : linear and angular velocity of frame $\{\mathrm{e}\}$ with respect to $\{i\}$ expresed in $\{a\}$

- ${ }_{a} t_{i}^{\mathrm{e}}=\left[{ }_{a} v_{i}^{\mathrm{e}},{ }_{a} w_{i}^{\mathrm{e}}\right]$ : twist of frame $\{\mathrm{e}\}$ with respect to $\{i\}$ expresed in $\{a\}$

- distances between the joint frames: $r=9 \mathrm{~mm}, 1=54.5 \mathrm{~mm}$, $a=3.5 \mathrm{~mm}, b=7.5 \mathrm{~mm}$ and $\mathrm{s}=30 \mathrm{~mm}$

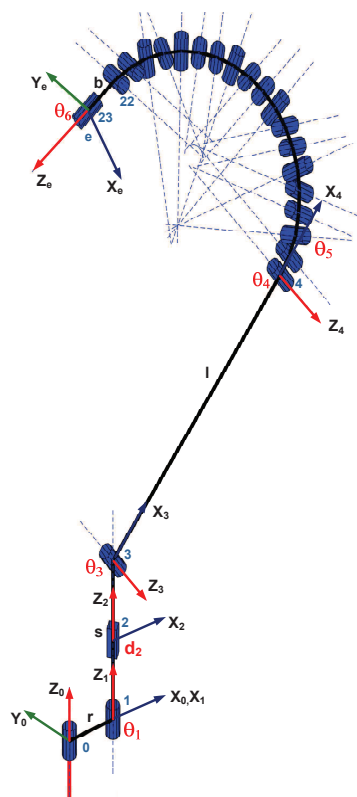

(a) Manipulator kinematics

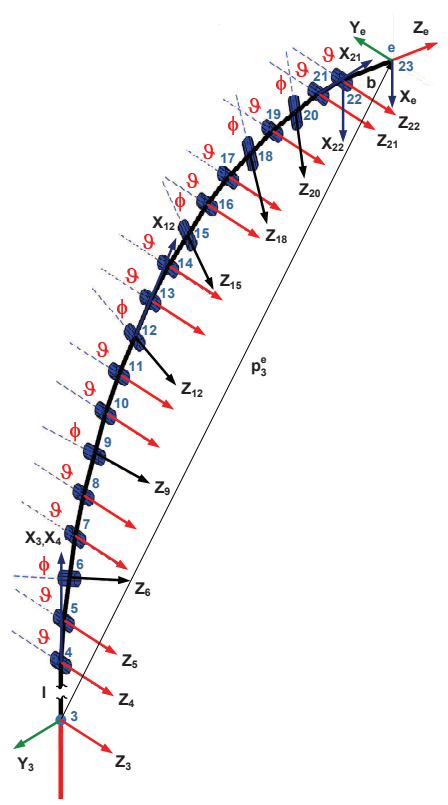

(b) Kinematics of the bending section
Fig. 5. Schematic drawing of the manipulator and the bending section kinematics generated with the robotic toolbox in Matlab. It illustrates the individual joints and the used coordinate axis.
TABLE II

DENAVIT-HARTENBERG TABLE OF THE SINGLE-PORT MANIPULATOR.

\begin{tabular}{|c|c|c|c|c|c|c|c|c|c|c|}
\hline$i$ & 1 & 2 & 3 & 4 & 5 & $\ldots$ & 20 & 21 & 22 & 23 \\
\hline$\alpha_{i}$ & 0 & 0 & $\frac{\pi}{2}$ & 0 & 0 & $\ldots$ & $\frac{\pi}{2}$ & $-\frac{\pi}{2}$ & 0 & $\frac{\pi}{2}$ \\
\hline$a_{i}$ & $\mathrm{r}$ & 0 & 0 & 1 & $\mathrm{a}$ & $\ldots$ & $\mathrm{a}$ & $\mathrm{a}$ & $\mathrm{a}$ & 0 \\
\hline$d_{i}$ & 0 & $d_{2}+\mathrm{s}$ & 0 & 0 & 0 & $\ldots$ & 0 & 0 & 0 & $\mathrm{~b}$ \\
\hline$\theta_{i}$ & $\theta_{1}$ & 0 & $\theta_{3}+\frac{\pi}{2}$ & $\vartheta$ & $\vartheta$ & $\ldots$ & $\phi$ & $\vartheta$ & $\vartheta-\frac{\pi}{2}$ & $\theta_{6}$ \\
\hline
\end{tabular}

The DH table of the manipulator is presented in Table II. All the passive hinges of the bending section are represented by joints 4 to 22 that corresponds to joints $\theta_{4}$ and $\theta_{5}$ of the manipulator. A complete joint data of the periodic hinge configuration of the bending section has already been published in [11]. The end-effector frame $\{\mathrm{e}\}$ is represented by the joint 23 that corresponds to joint $\theta_{6}$ of the manipulator.

\section{A. Forward Kinematics}

The forward kinematics of the single-port manipulator $T_{0}^{23}$ is computed using the transformation matrix $T_{0}^{3}$ for the first three joints, $T_{3}^{22}$ for the two joints of the bending section and $T_{22}^{23}$ for the rotation joint of the instrument at the tip.

$$
T_{0}^{23}=T_{0}^{3} \cdot T_{3}^{22} \cdot T_{22}^{23}
$$

The transformation matrix from base $\{0\}$ to the joint $\{3\}$ is:

$$
T_{0}^{3}=\left[\begin{array}{cccc}
-\cos \theta_{1} \sin \theta_{3} & -\cos \theta_{1} \cos \theta_{3} & \sin \theta_{1} & r \\
-\sin \theta_{1} \sin \theta_{3} & -\sin \theta_{1} \cos \theta_{3} & -\cos \theta_{1} & 0 \\
\cos \theta_{3} & -\sin \theta_{3} & 0 & d_{2}+s \\
0 & 0 & 0 & 1
\end{array}\right]
$$

The transformation matrix $T_{3}^{22}$, comprising the 19 passive joints of the bending section, is:

$$
T_{3}^{22}=T_{3}^{4} \cdot T_{4}^{21} \cdot T_{21}^{22}
$$

The transformation matrix $T_{4}^{21}$ is the forward kinematics of the bending section $T_{1}^{18}$ as presented in the equation (3) in [11] and the fist and last transformation matrix are:

$$
T_{3}^{4}=\left[\begin{array}{cccc}
\cos \vartheta & -\sin \vartheta & 0 & l \\
\sin \vartheta & \cos \vartheta & 0 & 0 \\
0 & 0 & 1 & 0 \\
0 & 0 & 0 & 1
\end{array}\right] \quad T_{21}^{22}=\left[\begin{array}{cccc}
\sin \vartheta & \cos \vartheta & 0 & a \\
-\cos \vartheta & \sin \vartheta & 0 & 0 \\
0 & 0 & 1 & 0 \\
0 & 0 & 0 & 1
\end{array}\right]
$$

The transformation from the last hinge of the bending section to the instrument frame $\{\mathrm{e}\}$ is:

$$
T_{22}^{23}=\left[\begin{array}{cccc}
\cos \left(\theta_{6}\right) & -\sin \left(\theta_{6}\right) & 1 & 0 \\
0 & 0 & 0 & b \\
-\sin \left(\theta_{6}\right) & -\cos \left(\theta_{6}\right) & 0 & 0 \\
0 & 0 & 0 & 1
\end{array}\right]
$$

\section{B. Instantaneous Kinematics}

The twist of the single-port manipulator is computed similar to the forward kinematics by summing three partial twists.

$$
{ }_{0} t_{0}^{23}={ }_{0} t_{0}^{3}+{ }_{0} t_{3}^{22}+{ }_{0} t_{22}^{23}
$$

The twist resulting from the first three joints of the manipulator is determined as follow: 


$$
\begin{gathered}
{ }_{0} t_{0}^{3}=\left[\begin{array}{lll}
J_{1} & J_{2} & J_{3}
\end{array}\right] \cdot\left[\begin{array}{lll}
\dot{\theta}_{1} & \dot{d}_{2} & \dot{\theta}_{3}
\end{array}\right]^{T} \\
\text { where } \quad J_{1}=\left[\begin{array}{c}
z_{1} \times\left(p_{0}^{\mathrm{e}}-p_{0}^{1}\right) \\
z_{1}
\end{array}\right] \\
J_{2}=\left[\begin{array}{c}
z_{2} \\
0
\end{array}\right] \quad \text { and } \quad J_{3}=\left[\begin{array}{c}
z_{3} \times\left(p_{0}^{\mathrm{e}}-p_{0}^{3}\right) \\
z_{3}
\end{array}\right]
\end{gathered}
$$

The twist of the bending section with respect to the joint $\{3\}$ is given by (4) in [11]. By using this equation, the twist with respect to the base $\{0\}$ is consequently:

$$
{ }_{0} t_{3}^{22}=E \cdot{ }_{3} t_{3}^{22}=E \cdot J_{D} \cdot \dot{\theta}_{D} \quad \text { where } \quad E=\left[\begin{array}{cc}
R_{0}^{3} & 0 \\
0 & R_{0}^{3}
\end{array}\right]
$$

According to the definition (6) in [11], by summing up the partial joint angles, the twist of the bending section with respect to the manipulator base is then:

$$
\begin{gathered}
{ }_{0} t_{3}^{22}=\dot{\vartheta} \cdot E \cdot \sum_{i=j}\left[\begin{array}{c}
z_{i} \times p_{i}^{\mathrm{e}} \\
z_{i}
\end{array}\right]+\dot{\phi} \cdot E \cdot \sum_{i=k}\left[\begin{array}{c}
z_{i} \times p_{i}^{\mathrm{e}} \\
z_{i}
\end{array}\right] \\
\text { where } \quad \begin{array}{l}
j=4,5,7,8,10,11,13,14,16,17,19,21,22 \\
k=6,9,12,15,18,20
\end{array}
\end{gathered}
$$

Hence, the Jacobians for the two individual joints $\theta_{4}$ and $\theta_{5}$ of the manipulator are:

$$
J_{4}=E \cdot \sum_{i=j}\left[\begin{array}{c}
z_{i} \times p_{i}^{\mathrm{e}} \\
z_{i}
\end{array}\right] \quad \text { and } \quad J_{5}=E \cdot \sum_{i=k}\left[\begin{array}{c}
z_{i} \times p_{i}^{\mathrm{e}} \\
z_{i}
\end{array}\right]
$$

and the twist of the bending section mapped to the manipulator base is:

$$
{ }_{0} t_{3}^{22}=\left[\begin{array}{ll}
J_{4} & J_{5}
\end{array}\right] \cdot\left[\begin{array}{ll}
\dot{\theta}_{4} & \dot{\theta}_{5}
\end{array}\right]^{T}
$$

The twist of the sixth joint (rotation of the instrument) is computed as follow:

$$
\begin{gathered}
{ }_{0} t_{22}^{23}=J_{6} \cdot \dot{\theta}_{6} \\
J_{6}=\left[\begin{array}{c}
z_{\mathrm{e}} \times\left(p_{0}^{\mathrm{e}}-p_{0}^{\mathrm{e}}\right) \\
z_{\mathrm{e}}
\end{array}\right]=\left[\begin{array}{c}
0 \\
R_{0}^{23} \cdot \hat{e}_{3}
\end{array}\right] \\
\text { where } \quad \hat{e}_{3}=\left[\begin{array}{lll}
0 & 0 & 1
\end{array}\right]^{T}
\end{gathered}
$$

Finally, the twist of the manipulator is given by the sum of the partial twists (7), (12) and (13).

$$
\begin{gathered}
0 t_{0}^{23}=\left[\begin{array}{l}
J_{v} \\
J_{w}
\end{array}\right] \cdot \dot{\theta}=J_{\mathrm{m}} \cdot \dot{\theta} \\
\text { where } \dot{\theta}=\left[\begin{array}{llllll}
\dot{\theta}_{1} & \dot{d}_{2} & \dot{\theta}_{3} & \dot{\theta}_{4} & \dot{\theta}_{5} & \dot{\theta}_{6}
\end{array}\right]^{T} \\
J_{\mathrm{m}}=\left[\begin{array}{llllll}
J_{1} & J_{2} & J_{3} & J_{4} & J_{5} & J_{6}
\end{array}\right]
\end{gathered}
$$

The $6 \times 6$ Jacobian $J_{\mathrm{m}}$ of the manipulator can be inverted to calculate the joint velocities $\dot{\theta}$ by a given twist of the end-effector.

\section{Velocity-Based Task-Space Control}

The control of the manipulators was implemented in a simulation environment that enables the parallel evaluation of the hardware development and system functionalities. The simulation of the complete surgical scenario, with the singleport manipulator attached to the SoloAssist and mounted on an operating table, was implemented using the Coin3D open source library. As shown in Fig. 6, the model of the singleport platform comprises a simplified one-to-one replica of the physical system. The simulation of the bowden wires and their friction is not implemented in the current version. A collision of the manipulators in the implemented simulation is avoided by using the supplied collision detection in the Coin3D library. This feature is also used, for example, for the detection of the interaction between the instruments and the objects that have to be manipulated. The workspace of the manipulators, kinematic structure of the platform and motion modalities were evaluated with this simulation.
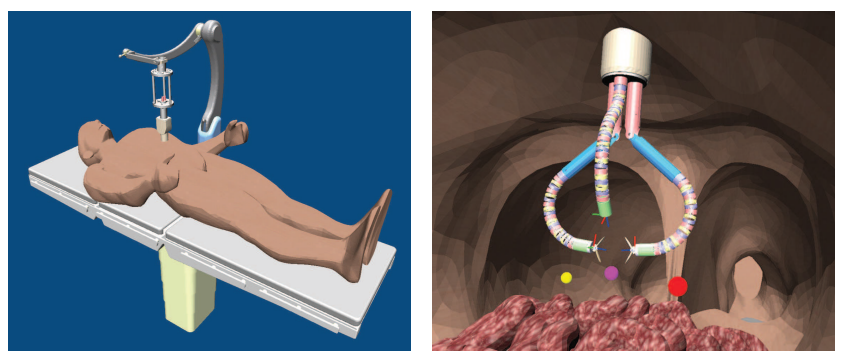

(a) External view of the simulation (b) Transabdominal view presenting with the complete surgical scenario the distal section of the platform

Fig. 6. Simulation environment of the single-port system for the evaluation of the control, kinematics and workspace analysis.

\section{A. Control Architecture of the Single-Port System}

A distributed, modular control architecture, as shown in Fig. 7, was conceived to control the single-port system. The high-level control forms the central control unit for all other entities. It gets, for example, the reference position from the master console and provides, after a path planning, the desired position and control mode for the low-level control. The real tool or joint position from the low-level control is the provided interface for the robot simulation. The current architecture implements the high-level and low-level control,

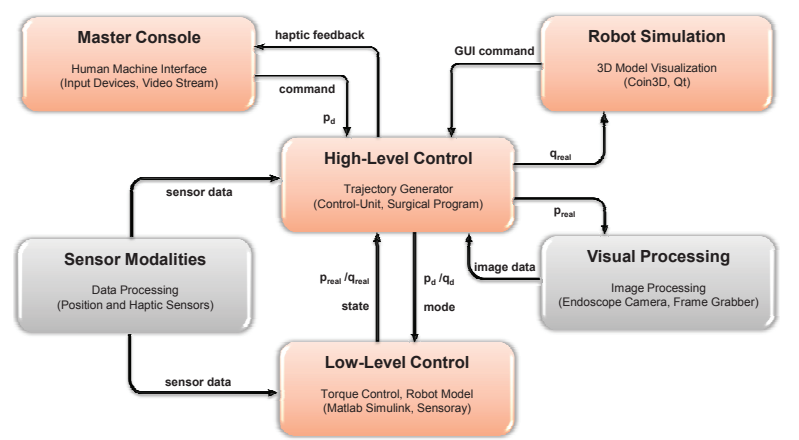

Fig. 7. Implemented modular control architecture of the single-port system. 
the master console for human machine interaction and the simulation of the robot model. Furthermore, it is conceived that the high-level control also processes the GUI commands from the simulation as well as sensor and image data.

Matlab-Simulink is used to implement the real-time lowlevel control of the manipulators. A Linux kernel with RTAI real-time extension is used for the control of the motor drives. Four motors are individually controlled over one Sensoray 626 I/O card. The encoder signals are fed directly over these cards to the computer, and a current control is realized with PWM amplifiers. The implemented control provides a UDP interface for the higher-level control over the network.

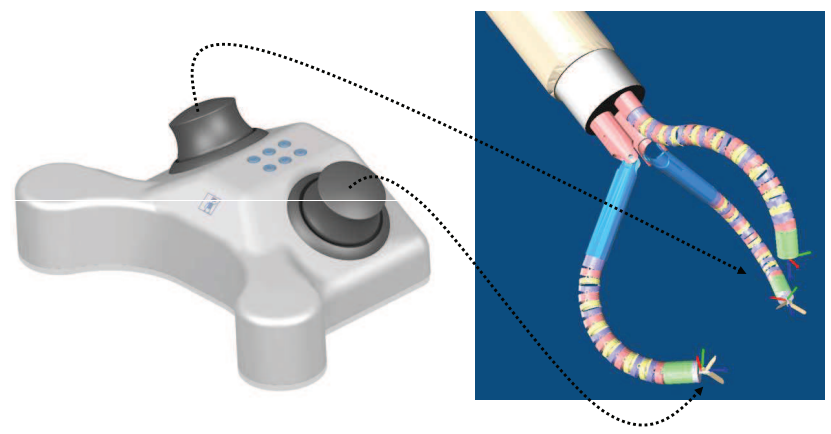

Fig. 8. Task-space control of the manipulators and the telescope with two 6 DOF space-mouse integrated in an input device.

\section{B. Task-Space Control of the Manipulators}

As illustrated in Fig. 8, two SpaceNavigator from 3Dconnexion are integrated into an input device to control the single-port manipulators. The 6 DOF position and orientation of the instrument tip and the grasping function are operated by using the space-mouse. Additionally six button switches are integrated into the input device that are used, for example, to exchange between the control of the manipulators and the telescope or the SoloAssist telemanipulator. These buttons are also used to switch between the different views in the simulation as well as to open and close both instruments.

A common velocity-based control, as shown in Fig. 9, was implemented in a model-view-controller paradigm to evaluate the task-space control of the implemented simulation. For the telemanipulation scenario, the displacement of the spacemouse is implemented as velocity vector that is multiplied with a gain factor and used as twist of the instrument. The joint velocities are computed by multiplying the twist with the inverse Jacobian of the manipulator. After integrating the joint velocities, the position difference of the instrument is controlled in the simulation.

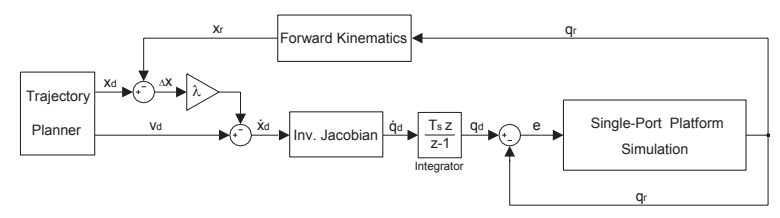

Fig. 9. Velocity-based control of the single-port manipulator. A basic velocity profile is used to control the simulation of the platform.
A simplified trajectory generator for a straight point to point control has also been implemented to realize a position control of the manipulators. In the current state, the position of the instruments can be controlled through a GUI command in the simulation. The trajectory is calculated in a thread with a cycle of $1 \mathrm{~ms}$ to minimize the discrete error resulting from the calculation. This error is also considered in each cycle by adding it to the calculated new position.

\section{Evaluation And Workspace Analysis}

\section{A. Pick-and-Place Scenario for Evaluation of the Control}

A pick-and-place scenario was programmed for the purpose of training and teaching the physicians. In this training exercise, surgeons had to grasp spheres with the instruments and place them at a predefined position. The evaluation includes the handover of the spheres from one manipulator to the other, the control of the entire platform to different quadrants of the abdomen and the adjustment of the endoscopic view by the control of the telescope.

Five physicians accomplished this evaluation to determine a qualitative assessment of the control and the input device. All experiments were carried out successfully, however, the position control with the space-mouse was challenging and unintuitive. The individual tasks were elaborate and required longer than expected. These experiments enabled also the verification of the implemented kinematics that resulted the controllability of the manipulators in the intended workspace.

\section{B. Workspace Analysis of the Manipulators}

We analyzed the theoretical workspace and manipulability of the manipulators in order to assess the capabilities and limitations of the system. The theoretical workspace that is obtained by deflection of the elbow joint by $\pm 90^{\circ}$ and the bending section by $\pm 180^{\circ}$ in the horizontal plane is presented in Fig. 10. The dark-red range shows the singular configuration of the manipulator where the determinant of the Jacobian is zero and the rank of the matrix is reduced. It is apparent that one of the singularity is in straight configuration of the manipulator where the rotation of the manipulator $\theta_{1}$ and the rotation of the instrument $\theta_{6}$ are overlapping.

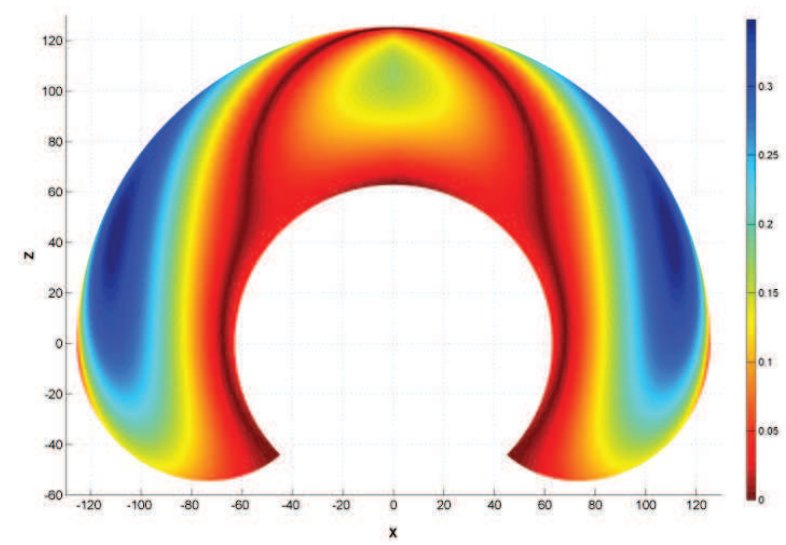

Fig. 10. Horizontal workspace and manipulability of the manipulator achieved by the deflection of the elbow joint and the bending section. 


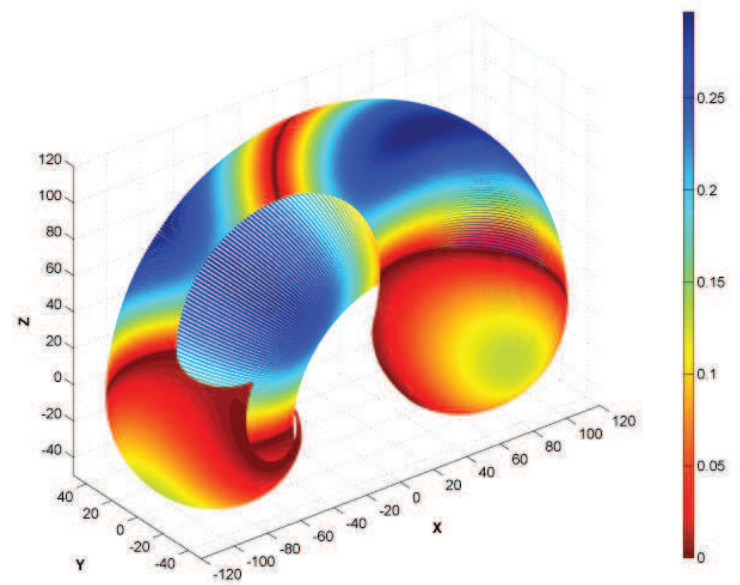

Fig. 11. Workspace and manipulability of the 3 distal manipulator joints.

The tridimensional workspace and manipulability of the three distal DOFs is shown in Fig. 11, which also includes the deflection of the bending section in the vertical plane. The illustrated surface is determined by the combination of three partial surfaces that presents the individual maximum working ranges. This includes the surface, which is achieved by horizontal deflection of the elbow joint by $\pm 90^{\circ}$ and vertical deflection of the bending section by $\pm 150^{\circ}$, as well as both surfaces achieved by the deflection of the bending section vertically by $\pm 150^{\circ}$ and horizontally by $+180^{\circ}$ on the left side and $-180^{\circ}$ on the right side. This figure shows further distinctive singular configurations of the manipulator. These are arranged by a deflection of the elbow at + or $-90^{\circ}$ and the bending section at $0^{\circ}$ as well as at + or $-180^{\circ}$.

The control of the manipulator through a singularity is achieved in the implemented simulation by using the pseudoinverse Jacobian. However, the stability and control behavior is not satisfactory in all cases, so that specific treatment of the singularities is necessary.

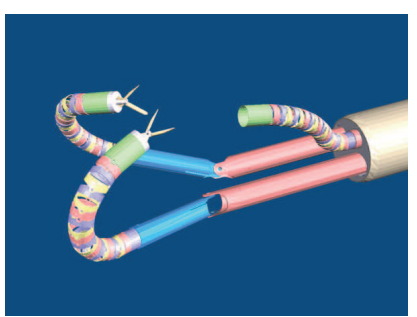

(a) Manipulator in retroflexion

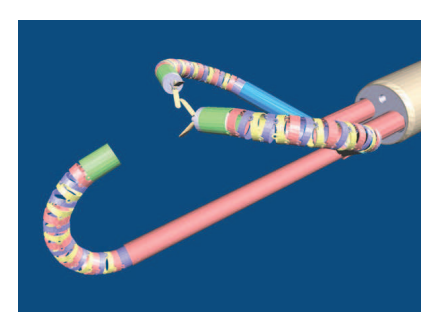

(b) Telescope in retroflexion
Fig. 12. Analysis of the working scenarios: retroflexed configuration of the manipulators or the telescope.

\section{Configuration Analysis and Application Scenarios}

In a typical surgical scenario the manipulators as well as the telescope are operated in prograde configuration. As illustrated in Fig. 12 the developed manipulators and the telescope provide an operation in retroflexion. This enables for example a surgical intervention on the rear side of an organ. Hence, the single-port system offers completely new possibilities in terms of hitherto not feasible surgical interventions. Additionally to the illustrated images, both the manipulators and the telescope could be operated in retroflexion. The control in all these three scenarios was unintuitive or even not possible and requires dedicated control solutions.

\section{CONCLUSION AND OUTLOOK}

We presented the current state of the developed singleport platform for minimally invasive surgery. The kinematics of the integrated $6 \mathrm{DOF}$ manipulators is also presented. By using the determined kinematics, a velocity-based task-space control was implemented for position control of the instruments. Moreover, the implemented control architecture and the task-space control with a space-mouse is described. The kinematics of the manipulator was verified and the implemented control was evaluated in a pick-and-place scenario. A workspace analysis was likewise performed to evaluate the theoretical capabilities and effectiveness of the system. Further studies will include the integration of an intuitive input device, the treatment of the manipulator singularities in the control and further analysis regarding the operation with the manipulators in retroflexed configuration.

\section{ACKNOWLEDGMENTS}

This work was supported by the funding of the research group 1321 from the German Research Foundation (DFG).

\section{REFERENCES}

[1] H. Feussner, S. Can, A. Fiolka, and A. Schneider, "Hybrid surgery-the way towards notes the challenge for computer science," in Biomedical Imaging: From Nano to Macro, 2008. ISBI 2008. 5th IEEE International Symposium on. IEEE, 2008, pp. 1383-1386.

[2] I. Gill, A. Advincula, M. Aron, J. Caddedu, D. Canes, P. Curcillo, M. Desai, J. Evanko, T. Falcone, V. Fazio, et al., "Consensus statement of the consortium for laparoendoscopic single-site surgery," Surgical endoscopy, vol. 24, no. 4, pp. 762-768, 2010.

[3] J. Romanelli and D. Earle, "Single-port laparoscopic surgery: an overview," Surgical endoscopy, vol. 23, no. 7, pp. 1419-1427, 2009.

[4] A. Pryor, J. Tushar, and L. DiBernardo, "Single-port cholecystectomy with the TransEnterix SPIDER: simple and safe," Surgical endoscopy, vol. 24, no. 4, pp. 917-923, 2010.

[5] J. Kaouk, R. Goel, G. Haber, S. Crouzet, and R. Stein, "Robotic single-port transumbilical surgery in humans: initial report," $B J U$ international, vol. 103, no. 3, pp. 366-369, 2009.

[6] G. Rao, M. Mansard, P. Ravula, P. Rebala, R. Dama, and D. Reddy, "Single-port surgery: Current applications and limitations," Asian Journal of Endoscopic Surgery, vol. 2, no. 3, pp. 56-64, 2009.

[7] D. Larkin, T. Cooper, C. Mohr, and D. Rosa, "Minimally invasive surgical instrument advancement," June 13 2007, uS Patent App. $11 / 762,161$.

[8] G. Haber, M. White, R. Autorino, P. Escobar, M. Kroh, S. Chalikonda, R. Khanna, S. Forest, B. Yang, et al., "Novel robotic da vinci instruments for laparoendoscopic single-site surgery," Urology, 2010.

[9] M. Piccigallo, U. Scarfogliero, C. Quaglia, G. Petroni, P. Valdastri, A. Menciassi, and P. Dario, "Design of a novel bimanual robotic system for single-port laparoscopy," Mechatronics, IEEE/ASME Transactions on, vol. 15, no. 6, pp. 871-878, 2010.

[10] J. Ding, K. Xu, R. Goldman, P. Allen, D. Fowler, and N. Simaan, "Design, simulation and evaluation of kinematic alternatives for insertable robotic effectors platforms in single port access surgery," in Robotics and Automation (ICRA), 2010 IEEE International Conference on. IEEE, pp. 1053-1058.

[11] S. Can, C. Staub, A. Knoll, A. Fiolka, A. Schneider, and H. Feussner, "Design, Development and Evaluation of a Highly Versatile Robot Platform for Minimally Invasive Single-Port Surgery." in Proceedings of the Fourth IEEE RAS/EMBS International Conference on Biomedical Robotics and Biomechatronics, 2012, pp. 817-822. 